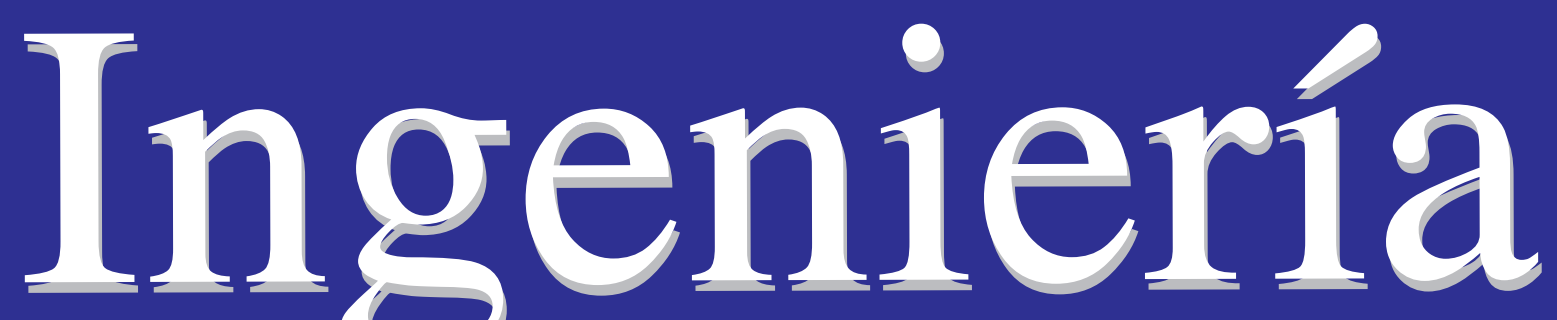

Revista de la Universidad de Costa Rica ENERO/ JULIO 2011 - VOLUMEN 21 Número (1)

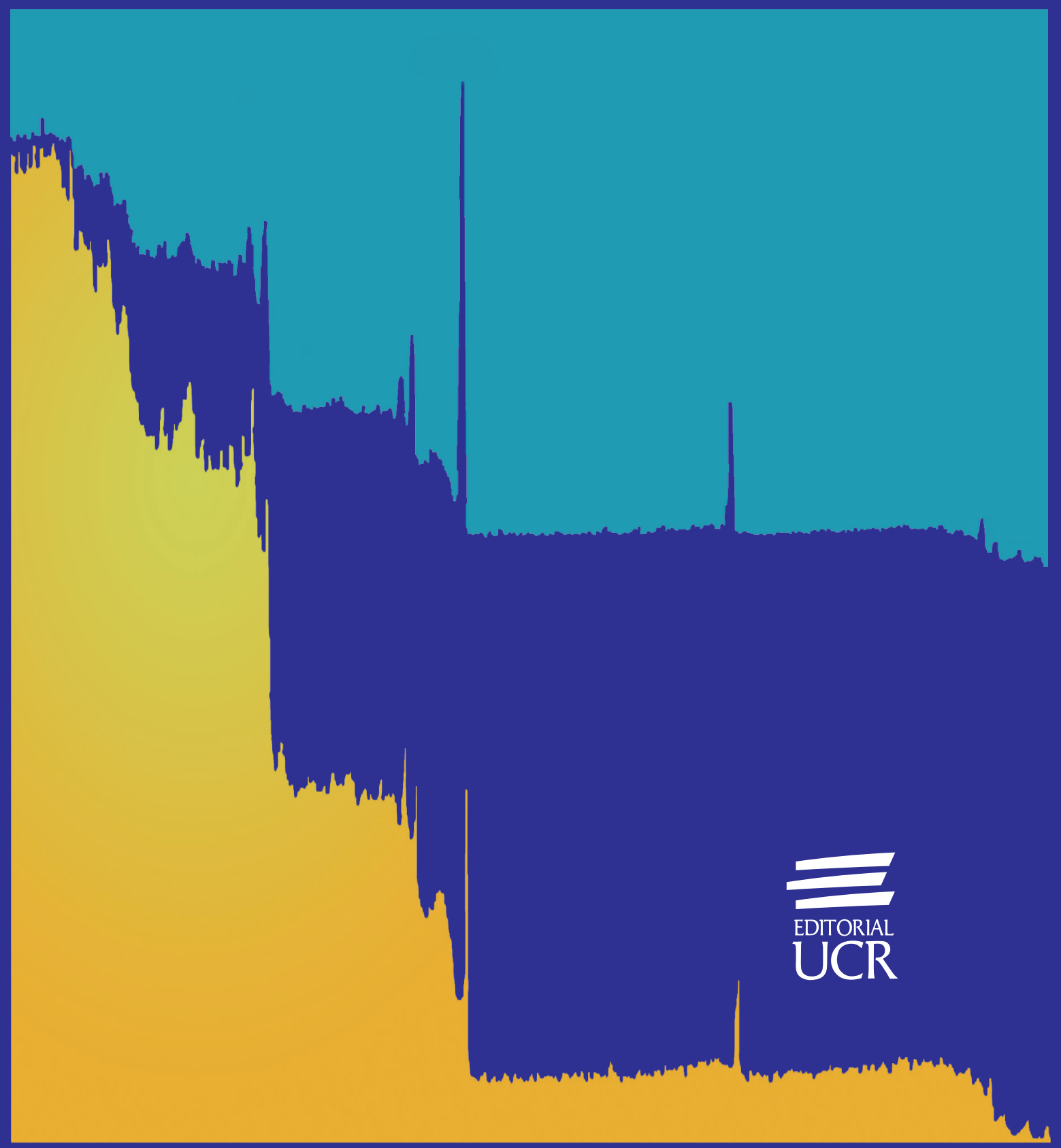




\title{
EVALUACIÓN DEL EFECTO DE LA TEMPERATURA, CONCENTRACIÓN Y FLUJO VOLUMÉTRICO EN LA HIDRÓLISIS DE SACAROSA MEDIANTE UNA INVERTASA INMOVILIZADA EN UN REACTOR ESFÉRICO
}

\section{María Monserrath Zamora Leitón Manuel Molina Córdoba Gerardo Chacón Valle}

\section{Resumen}

Se evaluó a nivel de laboratorio el efecto del flujo volumétrico, la temperatura y la concentración inicial de sacarosa, en la reacción de hidrólisis de sacarosa utilizando una invertasa inmovilizada. Ésta se inmovilizó en $20 \mathrm{~g}$ de soporte de tamaño de malla entre 120 y 140 . Se obtiene que la cantidad máxima de enzima inmovilizada es de $0,130 \mathrm{mg} / \mathrm{g}$ de soporte a los $220 \mathrm{~min}$.

La primera etapa experimental consistió en la evaluación del efecto de la concentración inicial de sacarosa $(1,0$ y $1,5 \mathrm{~mol} / \mathrm{L})$, el flujo volumétrico $(3,0 \mathrm{~mL} / \mathrm{min}$ y $4,0 \mathrm{~mL} / \mathrm{min})$ y la temperatura de reacción $\left(45^{\circ} \mathrm{C}\right.$ y $\left.50^{\circ} \mathrm{C}\right)$. Se encontró que el efecto de las tres variables anteriores son estadisticamente significativos. La conversión se favorece para una concentración $1,0 \mathrm{~mol} / \mathrm{L}$ de sacarosa, un flujo volumétrico de $3 \mathrm{~mL} / \mathrm{min}$ y una temperatura de $50^{\circ} \mathrm{C}$. La máxima conversión que se obtuvo fue de $95,4 \%$.

En una segunda etapa experimental se evaluó el efecto de la concentración inicial de sacarosa $(0,75$ y 1,0 mol/L), el flujo volumétrico $(2,5 \mathrm{~mL} / \mathrm{min}$ y $3,0 \mathrm{~mL} / \mathrm{min})$ y la temperatura $\left(50^{\circ} \mathrm{C}\right.$ y $\left.55^{\circ} \mathrm{C}\right)$. Se encontró que el flujo volumétrico y la interacción concentración de sacarosa - temperatura son significativas estadísticamente. La conversión se favorece para un flujo de $2,5 \mathrm{~mL} / \mathrm{min}$, y es preferible operar a $50^{\circ} \mathrm{C}$ y a una concentración $1,0 \mathrm{~mol} / \mathrm{L}$ de sacarosa. El mayor porcentaje de conversión de sacarosa fue de un $94,8 \%$.

En una última fase experimental, se analizó el efecto de la concentración inicial de sacarosa, se encontró que a una concentración 1,1 mol/L se obtiene el mayor porcentaje de conversión de un 95,0 \%, para una temperatura de $50^{\circ} \mathrm{C}$ y un flujo volumétrico de $2,5 \mathrm{~mL} / \mathrm{min}$.

Palabras clave: hidrólisis de sacarosa, inmovilización enzimática, invertasa, reactor esférico

\begin{abstract}
This research evaluated the effect of the volumetric flow, the temperature and the initial concentration of sucrose in the reaction of hydrolysis of sucrose by immobilized invertase, in the laboratory. Invertase was immobilized in $20 \mathrm{~g}$ of support size mesh between 120 and 140. The maximum quantity of immobilized invertase obtained was $0,130 \mathrm{mg} / \mathrm{g}$ of support at $220 \mathrm{~min}$.

In the first experimental stage, the effect of the initial concentration of sucrose $(1,0$ and $1,5 \mathrm{~mol} / \mathrm{L})$, the volumetric flow $(3,0 \mathrm{~mL} / \mathrm{min}$ y $4,0 \mathrm{~mL} / \mathrm{min})$ and the temperature $\left(45^{\circ} \mathrm{C}\right.$ y $\left.50^{\circ} \mathrm{C}\right)$ were evaluated. The effect of the above three variables was statistically significant. The conversion was favorable for a concentration of sucrose $1,0 \mathrm{~mol} / \mathrm{L}$, a volumetric flow of $3 \mathrm{~mL} / \mathrm{min}$ and a temperature of $50^{\circ} \mathrm{C}$. The maximum conversion obtained was $95,4 \%$.

The second experimental stage analyzed the effect of the initial concentration of sucrose $(0,75$ and $1,0 \mathrm{~mol} / \mathrm{L})$, the volumetric flow $(2,5 \mathrm{~mL} / \mathrm{min}$ and $3,0 \mathrm{~mL} / \mathrm{min})$ and the temperature $\left(50^{\circ} \mathrm{C}\right.$ and $\left.55^{\circ} \mathrm{C}\right)$. The variable of volumetric flow and the interaction concentration of sucrose - temperature were statistically significant. The conversion was favorable for a volumetric flow of $2,5 \mathrm{~mL} / \mathrm{min}$, and it was preferable to work at a temperature of $50^{\circ} \mathrm{C}$ with an initial concentration of sucrose of $1,0 \mathrm{~mol} / \mathrm{L}$. The maximum conversion was $94,8 \%$.

In the last experimental stage it was analyzed the effect of the concentration, it was found that the maximum conversion percentage was $95,0 \%$ for a concentration of $1,1 \mathrm{~mol} / \mathrm{L}$, for a temperature of $50^{\circ} \mathrm{C}$ and for a volumetric flow of $2,5 \mathrm{~mL} / \mathrm{min}$.
\end{abstract}

Keywords: Hydrolysis of sucrose, immobilization of enzymes, invertase, spherical reactor

Recibido: 11 de junio de $2010 \cdot$ Aprobado: 21 de setiembre de 2010 


\section{INTRODUCCIÓN}

En la actualidad existen diferentes tipos de edulcorantes, algunos de estos se encuentran de forma natural en los alimentos, tal es el caso de la sacarosa. Este edulcorante se agrega a diferentes tipos de alimentos, bebidas y jarabes.

Muchas de las empresas que utilizan la sacarosa como edulcorante, deben tratarla antes de emplearla como materia prima, ya que se requiere la eliminación de ciertas sustancias no deseadas, por lo que deben solubilizarla. El proceso de purificación implica un gasto más en el proceso, por lo que es más económico usar un edulcorante como materia prima que ya se encuentre en estado líquido y que no presente impurezas.

La sacarosa es un disacárido de glucosa y fructosa que por medio de una reacción de hidrólisis se descompone en estas sustancias, para obtener como producto el azúcar invertido. Este es un edulcorante líquido utilizado en la industria de alimentos; presenta un mayor poder edulcorante y una menor tendencia a cristalizar, en comparación a la sacarosa, por lo que le confiere más estabilidad, color y sabor al producto (Herrera, 2003).

La enzima invertasa, $\beta$-D-fructofuranosidasa, es el agente catalítico en la reacción de hidrólisis de sacarosa. Ésta proviene de cepas especiales de levadura Saccharomyces cerevisiae y el pH óptimo de inversión es de 4,5. La máxima actividad enzimática se presenta a temperaturas entre $50^{\circ} \mathrm{C}-60^{\circ} \mathrm{C}$ (Herrera, 2003).

Generalmente las enzimas son más estables térmicamente si son inmovilizadas, esta estabilidad tiene como consecuencia un incremento en la actividad enzimática; razón por la cual se requiere la inmovilización de la enzima en un soporte, tal como se realizó en esta investigación. La inmovilización permite su aplicación en sistemas continuos como en un reactor esférico, con la ventaja adicional de su reutilización (Illanes, 1994; Ortega, 1993; Woodward, 1985).

El reactor esférico de lecho empacado, se caracteriza porque su construcción no es costosa, minimiza la caída de presión y es la forma más económica para altas presiones. Conforme el flujo de alimentación avanza por el reactor y entra en la esfera, se produce un aumento en el área de sección transversal, de manera que la velocidad másica superficial disminuye, como consecuencia la caída de presión hidráulica se reduce. Como el área de sección transversal es pequeña cerca de la entrada y salida del reactor, la presencia de catalizador en estas secciones, podría provocar una caída de presión apreciable, lo cual disminuye la eficiencia del reactor. Por esta razón, se coloca una malla para retener el catalizador, tanto en las cercanías de la entrada como de la salida de la esfera (Fogler, 2001).

La producción de azúcar invertido por medio de la invertasa a partir de soluciones de sacarosa de caña de azúcar, es un tema que se ha estudiado con anterioridad, pero, en dichos estudios no se han realizado ensayos con un reactor esférico. Ésta es una de las razones por las cuales se realizó esta investigación.

Amaya et al (2006), presentan un estudio sobre la hidrólisis de sacarosa por medio de invertasa inmovilizada en nylon-6 empleando glutaraldehído, para tal fin se utilizó un reactor tubular. La temperatura óptima que se obtuvo fue de $60^{\circ} \mathrm{C}$ y se evaluó el efecto de tres flujos volumétricos y dos diferentes concentraciones de sacarosa. Se determinaron conversiones del $95 \%$ y $97 \%$, para concentraciones de sacarosa mayores a $2 \mathrm{~mol} / \mathrm{L}$.

Soto (2007), realizó la hidrólisis de sacarosa en un reactor tubular de lecho empacado por medio de la invertasa. Se determinó el efecto del flujo volumétrico y el tamaño de soporte, y se obtuvo un porcentaje de conversión máximo del $91 \%$, para una temperatura de $50^{\circ} \mathrm{C}$, una concentración de sacarosa $1 \mathrm{~mol} / \mathrm{L}$, un soporte de malla 140 y un flujo volumétrico de $1 \mathrm{~mL} / \mathrm{min}$.

El objetivo de esta investigación fue el evaluar a nivel de laboratorio el efecto del flujo volumétrico, la temperatura y la concentración inicial de sacarosa, en la reacción de hidrólisis de sacarosa por medio de la enzima invertasa inmovilizada, en un reactor esférico.

\section{MATERIALES Y MÉTODOS}

\subsection{Enzima invertasa}

La enzima invertasa que se utilizó es de la marca Novozymes (Novozymes, 2002). 


\subsection{Sacarosa}

Se utilizó azúcar comercial, adquirida en un mercado local, para la preparación de las diferentes soluciones de sacarosa.

\subsection{Inmovilización enzimática}

Para la inmovilización se emplean "pellets" de soporte que previamente se molieron en un molino de cuchillas y posteriormente se tamizaron. Se selecciona el tamaño de partículas entre las mallas 120 y 140 , se escoge este tamaño ya que entre más pequeña sea la partícula más cantidad de enzima se inmoviliza, ya que existe mayor superficie de contacto, en comparación a partículas de mayor tamaño (Ortega, 1993).

De estas partículas se toman $20 \mathrm{~g}$ para llevar a cabo la inmovilización, dicha cantidad es la que se requiere para llenar el espacio entre las mallas del reactor. La técnica de inmovilización que se usa es el entrecruzamiento, el procedimiento se puede consultar en Salleh (1982) y Montes (2004).

El soporte se activa previamente con glutaraldehído y se usa polietilenimina (PEI) como espaciador, para inmovilizar la invertasa (Amaya et al, 2006).

La cantidad de enzima inmovilizada es igual a la diferencia de concentración de proteína en el tiempo cero y al finalizar la inmovilización. La enzima inmovilizada se guarda en refrigeración en un buffer de acetatos de pH 5,0.

\subsection{Equipo experimental}

En la Figura 1 se presenta el diagrama del equipo que se utilizó para llevar a cabo la reacción de hidrólisis de sacarosa en un proceso continuo.

Se emplea un baño térmico con agua para mantener constante la temperatura de la solución de sacarosa. Con el mismo fin se coloca un aislante de fibra de vidrio en la superficie del reactor. $\mathrm{La}$ solución se alimenta al reactor esférico por medio de una bomba peristáltica previamente calibrada. A la salida del reactor se coloca una válvula para regular el flujo, de tal manera que el flujo de salida sea igual al de entrada.

El reactor tiene un diámetro interno de 5,30 $\mathrm{cm}$ para un volumen total de $0,8 \mathrm{~L}$. A la entrada y salida del mismo se colocan mallas de acero inoxidable número 200 (ver Figura 1) para retener la invertasa.

\subsection{Métodos de análisis}

Cuantificación de proteínas: se utiliza el método de Bradford (1976) para determinar la cantidad de proteína enzimática inmovilizada en el soporte. Se lee la absorbancia con un espectrofotómetro Spectronic $20 \mathrm{D}$, a una longitud de onda de $595 \mathrm{~nm}$.

Cuantificación de azúcares reductores: para determinar la cantidad de azúcares reductores a la salida del reactor se usa el método de NelsonSomoghi descrito por Southgate (1976). Por medio

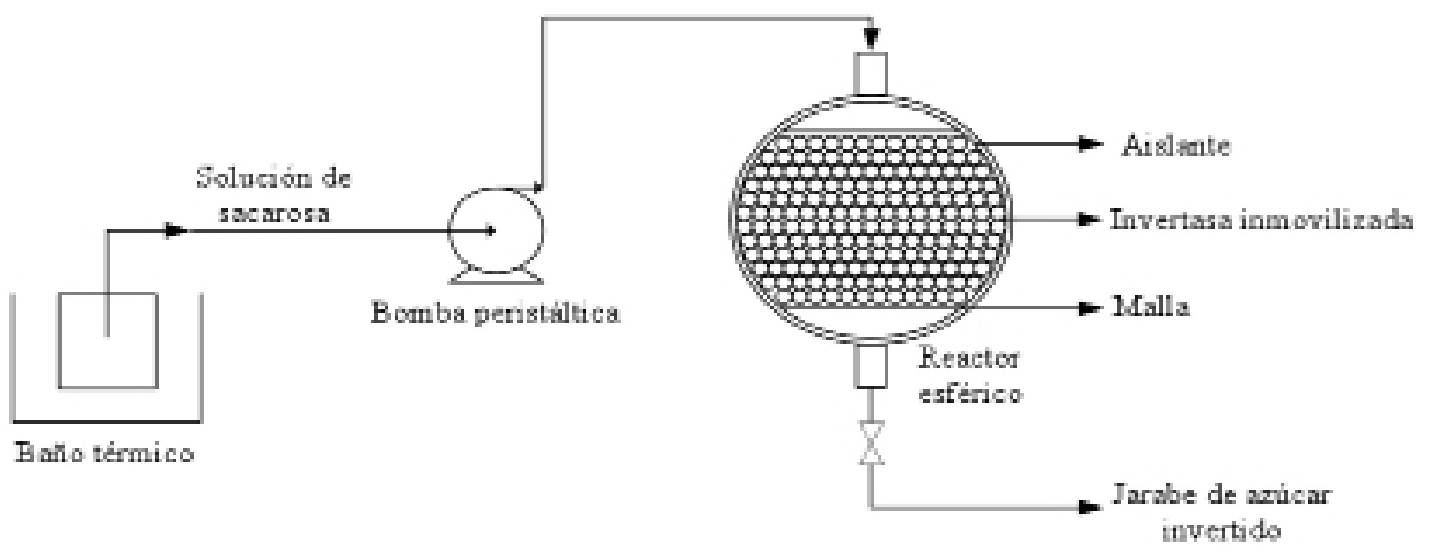

Figura 1. Equipo utilizado en la hidrólisis de sacarosa Fuente: (La autora y los autores) 
de un espectrofotómetro Spectronic $20 \mathrm{D}$, se lee la absorbancia a una longitud de onda de $500 \mathrm{~nm}$.

\subsection{Efecto de la concentración inicial de sacarosa, del flujo volumétrico y de la temperatura,primera etapa experimental}

El objetivo de esta etapa es evaluar el efecto del flujo volumétrico, la temperatura y la concentración inicial de sacarosa, en la reacción de hidrólisis de sacarosa por medio de la enzima invertasa inmovilizada, en un reactor esférico.

Las variables de interés, se estudian utilizando un diseño estadístico factorial $2^{3}$, en dos niveles, se repiten tres ensayos. Los niveles de las variables se fijaron a partir de la ficha técnica de la enzima y de fuentes bibliográficas.

Las variables fijas son el tamaño de las partículas de soporte el cual se encuentra entre las mallas 120 y 140; y el pH de la solución de sacarosa se fija en 4,5 con ácido cítrico.

Las variables experimentales son las siguientes: temperatura $\left(45^{\circ} \mathrm{C}\right.$ y $\left.50^{\circ} \mathrm{C}\right)$, concentración de la solución de sacarosa $(1,0 \mathrm{~mol} / \mathrm{L}$ y $1,5 \mathrm{~mol} / \mathrm{L})$ y el flujo volumétrico $(3,0 \mathrm{~mL} / \mathrm{min}$ y $4,0 \mathrm{~mL} /$ min). La variable medida es la concentración de azúcares reductores y la variable de respuesta es la conversión de sacarosa en azúcares reductores.

Inicialmente, se empaca el reactor con la enzima acoplada al soporte. Como dicho soporte se encontraba en un buffer de acetato de $\mathrm{pH} 5,0$ se hace fluir abundante agua destilada por el reactor para eliminar cualquier resto de la solución de acetato. Se prepara la solución de sacarosa de la concentración deseada y se regula el $\mathrm{pH}$. Se coloca en el baño térmico hasta que alcance la temperatura requerida para la reacción.

La solución se alimenta al reactor por medio de una bomba peristáltica, la válvula a la salida del reactor se mantiene cerrada hasta que el reactor se llene con la solución, en ese momento se regula el flujo de salida, para que sea igual al de entrada. Justo en ese instante se tiene el tiempo cero de la reacción y se toman muestras a la salida del reactor cada cierto tiempo para determinar el momento en que el sistema se encuentra en estado estable, es decir, cuando la concentración de azúcares reductores permanece constante.

A cada muestra que se toma se le agrega carbonato de calcio para detener la reacción, en caso de que alguna partícula de enzima haya atravesado la malla. Según pruebas preliminares se encontró que el estado estable se alcanza aproximadamente entre 2 horas y 2 horas con 30 minutos, por lo tanto cada ensayo se realiza en un periodo de 2 horas y 40 minutos.

Cuando el ensayo termina, el reactor se lava con abundante agua destilada para eliminar cualquier resto de azúcar, posteriormente se bombea buffer de acetato y se llena con este, hasta que sea utilizado nuevamente. El reactor con la enzima se almacenan en refrigeración.

\subsection{Efecto de la concentración inicial de saca- rosa, del flujo volumétrico y de la tempera- tura, segunda etapa experimental}

El objetivo de esta etapa, es evaluar a otros niveles el efecto del flujo volumétrico, la temperatura y la concentración inicial de sacarosa, en la reacción de hidrólisis de sacarosa por medio de la enzima invertasa inmovilizada.

Los ensayos se distribuyen en forma de un diseño factorial $2^{3}$, dos de ellos se repiten. Las variables fijas son el tamaño de las partículas de soporte el cual se encuentra entre las mallas 120 y 140; y el pH de la solución de sacarosa que se fija en 4,5 con ácido cítrico.

Las variables experimentales son las siguientes: la temperatura $\left(50^{\circ} \mathrm{C}\right.$ y $\left.55^{\circ} \mathrm{C}\right)$, la concentración de la solución de sacarosa $(0,75 \mathrm{~mol} / \mathrm{L}$ y $1,0 \mathrm{~mol} / \mathrm{L})$ y el flujo volumétrico $(2,5 \mathrm{~mL} / \mathrm{min}$ y $3,0 \mathrm{~mL} /$ min). La variable medida es la concentración de azúcares reductores y el porcentaje de conversión en azúcares reductores es la variable de respuesta.

Se sigue la misma metodología que se presenta en la sección 2.6.

\subsection{Efecto de la concentración inicial de sacarosa}

El objetivo es determinar cuál concentración inicial de sacarosa de las estudiadas favorece el porcentaje de conversión, para condiciones fijas de temperatura y flujo volumétrico, en la reacción de hidrólisis de sacarosa por medio de la enzima invertasa inmovilizada. Se seleccionan 
los valores de las variables según los resultados obtenidos en las etapas experimentales anteriores.

Las variables fijas son: el tamaño de las partículas de soporte (entre las mallas $120 \mathrm{y}$ 140), el $\mathrm{pH}$ de 4,5 , la temperatura de $50^{\circ} \mathrm{C}$ y el flujo volumétrico de $2,5 \mathrm{~mL} / \mathrm{min}$.

La variable experimental es la concentración de la solución de sacarosa: $0,75 \mathrm{~mol} / \mathrm{L}, 0,85 \mathrm{~mol} / \mathrm{L}$, $1,00 \mathrm{~mol} / \mathrm{L}, 1,10 \mathrm{~mol} / \mathrm{L}$ y $1,20 \mathrm{~mol} / \mathrm{L}$. La variable medida es la concentración de azúcares reductores y la variable de respuesta es la conversión de sacarosa en azúcares reductores.

La metodología que se utiliza es la que se presenta en la sección 2.6.

\section{RESULTADOS Y DISCUSIÓN}

\subsection{Inmovilización de la invertasa}

En la Figura 2 se muestra la cinética de inmovilización enzimática sobre el soporte; conforme aumenta el tiempo del proceso de inmovilización, se incrementa la cantidad de enzima inmovilizada, de manera que en la solución la cantidad de proteína es menor.

Como se observa en la figura anterior, la cantidad máxima de enzima inmovilizada es de $0,130 \mathrm{mg} / \mathrm{g}$ de soporte, esta se obtuvo a los 220 min; el proceso de inmovilización se mantuvo por 24 h para asegurar la inmovilización completa de la enzima en el soporte.

\subsection{Hidrólisis de sacarosa en un reactor esférico}

Esta fase se realizó en dos etapas experimentales, en cada una de ellas se analizó el efecto de la concentración inicial de sacarosa, del flujo volumétrico y de la temperatura, en la reacción de hidrólisis de sacarosa a diferentes niveles.

\subsubsection{Análisis de la primera etapa experimental}

En el Cuadro 1 se presentan los resultados de conversión de sacarosa en azúcares reductores obtenidos en el estado estable para cada uno de los ensayos y su réplica $\left(\mathrm{X}_{2}\right)$.
Según el Cuadro 1 la máxima conversión que se obtuvo en el estado estable es de un 95,43 $\%$, para una concentración inicial de sacarosa $1,0 \mathrm{~mol} / \mathrm{L}$ un flujo volumétrico de $4 \mathrm{~mL} / \mathrm{min}$ y una temperatura de $50^{\circ} \mathrm{C}$.

En el Cuadro 2 se presenta los resultados del análisis estadístico; se encuentra que para el intervalo de no confianza del $95 \%$, tanto la concentración de sacarosa, flujo volumétrico y temperatura son significativos, por el contrario ninguna interacción es significativa.

Del análisis de los resultados (Box et al, 1993) se encuentra que, la concentración de sacarosa disminuye en 19,3 unidades la conversión si ésta se incrementa, por lo que es conveniente trabajar a concentraciones más bajas de sustrato, en este caso una concentración $1,0 \mathrm{~mol} / \mathrm{L}$ de sacarosa. Esto se produce porque al disminuir la concentración de sustrato, la saturación enzimática es menor y decrece la posibilidad de que el producto se comporte como inhibidor. Si se utiliza concentraciones altas de sustrato permite la elaboración de jarabes más concentrados, lo cual es deseable para el producto, además se tiene que evaporar menos cantidad de agua para concentrar el sustrato. Al aumentar el flujo volumétrico de 3 $\mathrm{mL} / \mathrm{min}$ a $4 \mathrm{~mL} / \mathrm{min}$ se obtiene que el porcentaje de conversión disminuye en 16,3 unidades, por lo que es mejor emplear flujos más pequeños, ya que se aumenta el tiempo de residencia en el reactor. La solución de sacarosa tiene un tiempo de contacto mayor con la enzima lo cual favorece la reacción y en consecuencia el porcentaje de conversión de sacarosa en azúcares reductores aumenta.

Para el caso de la temperatura, si esta aumenta se beneficia la reacción, ya que al pasar de $45^{\circ} \mathrm{C}$ a $50^{\circ} \mathrm{C}$ se da un incremento de 20,6 unidades en la conversión; se favorece la actividad enzimática de la invertasa. Esto permite que el movimiento de las moléculas sea más rápido, de manera que es más probable que los movimientos aleatorios de estas las pongan en contacto con el sitio activo de la enzima. Este comportamiento está descrito por la ecuación de Arrhenius ya que un incremento en la temperatura favorece la constante específica de la reacción. Por lo tanto la reacción se acelera al aumentar la temperatura, pero se tiene que 


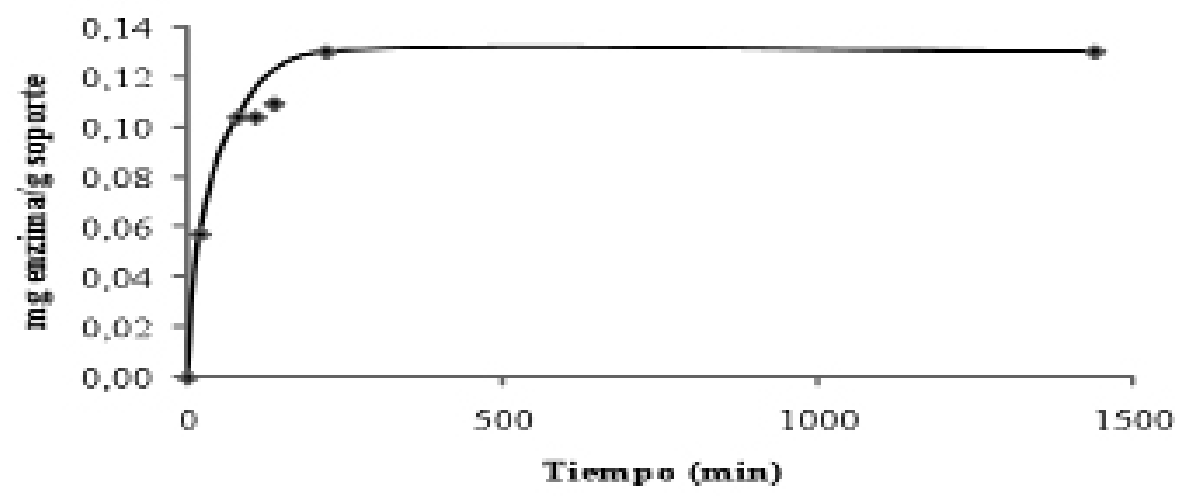

Figura 2. Cinética de inmovilización de la invertasa sobre partículas de soporte Fuente: (La autora y los autores)

Cuadro 1. Conversión de sacarosa en azúcares reductores

\begin{tabular}{|c|c|c|c|c|c|}
\hline Ensayo & $\begin{array}{c}\text { Concentración } \\
\text { sacarosa, } \\
\text { Cn } \\
\text { sacarosa }(\mathrm{mol} / \mathrm{L})\end{array}$ & $\begin{array}{l}\text { Flujo volumétri- } \\
\text { co, } F v(\mathrm{~mL} / \mathrm{min})\end{array}$ & $\begin{array}{c}\text { Temperatura, } \\
T\left({ }^{\circ} \mathrm{C}\right)\end{array}$ & $\begin{array}{c}\text { Conversión } \\
\text { de sacarosa, } \\
X_{1}(\%)\end{array}$ & $\begin{array}{c}\text { Conversión } \\
\text { de sacarosa, } \\
X_{2}(\%)\end{array}$ \\
\hline 1 & 1,0 & 3,0 & 45 & 80,14 & - \\
\hline 2 & 1,5 & 3,0 & 45 & 64,79 & 70,50 \\
\hline 3 & 1,0 & 4,0 & 45 & 59,99 & - \\
\hline 4 & 1,5 & 4,0 & 45 & 42,39 & - \\
\hline 5 & 1,0 & 3,0 & 50 & 94,56 & - \\
\hline 6 & 1,5 & 3,0 & 50 & 81,54 & - \\
\hline 7 & 1,0 & 4,0 & 50 & 95,04 & 95,43 \\
\hline 8 & 1,5 & 4,0 & 50 & 52,33 & 69,85 \\
\hline
\end{tabular}

Los ensayos 2,7 y 8 se replicaron.

Fuente: (La autora y los autores) 
Cuadro 2. Análisis estadístico para la variable de conversión de sacarosa en azúcares reductores, primera etapa experimental

\section{Estadístico}

Promedio, conversión de sacarosa (\%)

Efecto 1, concentración sacarosa, E1

Efecto 2, flujo volumétrico, E2

Interacción1-2, I12

Efecto 3, temperatura, E3

Interacción 1-3, I13

Interacción 2-3, I23

Interacción 1-2-3, I123

Promedio de los efectos

Varianza de los efectos, $\sigma^{2}$

Intervalo de no significancia (95\%)
Valor

72,82

$-19,31$

$-16,30$

$-6,56$

20,56

$-4,27$

6,41

$-4,01$

$-3,35$

20,61

$[-13,62 ; 13,62]$

Fuente: (La autora y los autores)

Cuadro 3. Conversión de sacarosa en azúcares reductores

\begin{tabular}{|c|c|c|c|c|c|}
\hline Ensayo & $\begin{array}{c}\text { Concentración } \\
\text { sacarosa, } \\
\text { Cn }_{\text {sacarosa }}(\mathrm{mol} / \mathrm{L})\end{array}$ & $\begin{array}{l}\text { Flujo volumétrico, } \\
\qquad F v(\mathrm{~mL} / \mathrm{min})\end{array}$ & $\begin{array}{c}\text { Temperatura, } \\
T\left(\left(^{\circ} \mathbf{C}\right)\right.\end{array}$ & $\begin{array}{c}\text { Conversión } \\
\text { de sacarosa, } \\
x_{1}(\%)\end{array}$ & $\begin{array}{c}\text { Conversión } \\
\text { de sacarosa, } \\
X_{2}(\%)\end{array}$ \\
\hline 1 & 0,75 & 2,5 & 50 & 75,31 & - \\
\hline 2 & 1,00 & 2,5 & 50 & 94,26 & 90,17 \\
\hline 3 & 0,75 & 3,0 & 50 & 66,22 & - \\
\hline 4 & 1,00 & 3,0 & 50 & 82,04 & - \\
\hline 5 & 0,75 & 2,5 & 55 & 91,67 & - \\
\hline 6 & 1,00 & 2,5 & 55 & 94,82 & - \\
\hline 7 & 0,75 & 3,0 & 55 & 91,86 & - \\
\hline 8 & 1,00 & 3,0 & 55 & 87,16 & 80,46 \\
\hline
\end{tabular}

Los ensayos 2 y 8 se replican Fuente: (La autora y los autores) 
controlar la misma, ya que si se eleva en demasía puede ocurrir la desnaturalización de la enzima (Audesirk y Audesirk, 1996).

\subsubsection{Análisis de la segunda etapa experimental}

En el Cuadro 3 se presentan los resultados de conversión de sacarosa en azúcares reductores obtenidos en el estado estable para cada uno de los ensayos y su réplica.

Del Cuadro 3 se tiene que el mayor porcentaje de conversión de sacarosa en azúcares reductores es de un $94,82 \%$ para una temperatura de $55^{\circ} \mathrm{C}$, un flujo volumétrico de $2,5 \mathrm{~mL} / \mathrm{min}$ y una concentración inicial de sacarosa $1,0 \mathrm{~mol} / \mathrm{L}$.

En el Cuadro 4 se presenta los efectos e interacciones; el análisis estadístico muestra que para el intervalo de no significancia los efectos flujo volumétrico (E2), la temperatura (E3) y la interacción concentración de sacarosa - temperatura (I13) son significativos ya que se encuentran fuera del intervalo de no significancia.

En la Figura 3 se presenta el gráfico t' Student para los efectos e interacciones ordenados, para esta segunda etapa. Se encontró que el intervalo de no significancia para un $95 \%$ de confianza es de $[-7,44 ; 7,44]$, se corrobora que los efectos significativos son el flujo volumétrico (E2), la temperatura (E3) y la interacción concentración de sacarosa - temperatura (I13).

De los resultados se encuentra que para el flujo volumétrico se presenta una disminución de 7,5 unidades en el porcentaje de conversión al incrementarse el flujo de $2,5 \mathrm{~mL} / \mathrm{min}$ a 3,0 $\mathrm{mL} / \mathrm{min}$, de manera que es mejor emplear flujos volumétricos menores para aumentar el tiempo de retención dentro del reactor y favorecer el contacto del sustrato con la enzima. Esta tendencia se obtuvo también en la primera etapa experimental.

Se tiene que el efecto combinado de las variables de concentración inicial de sustrato y de temperatura, produce una disminución de 9,4 unidades en el porcentaje de conversión, pero como existe una interacción entre dichas variables, su efecto sobre la conversión se debe analizar con una tabla de doble entrada. Se obtiene estadísticamente que al utilizar el nivel alto de temperatura y el nivel bajo de concentración de sacarosa se favorece la conversión, ya que se obtiene un valor del 91,77 \% y este es el más alto.

Al mantener fijo el nivel bajo de concentración, ya sea en $0,75 \mathrm{~mol} / \mathrm{L}$ ó $1,0 \mathrm{~mol} / \mathrm{L}$ e incrementar la temperatura de $50^{\circ} \mathrm{C}$ a $55^{\circ} \mathrm{C}$ se da un aumento en la conversión, por lo que elevar la temperatura favorece la variable de respuesta.

Físicamente hay que tomar en cuenta que operar a temperaturas más altas de $55^{\circ} \mathrm{C}$ puede provocar

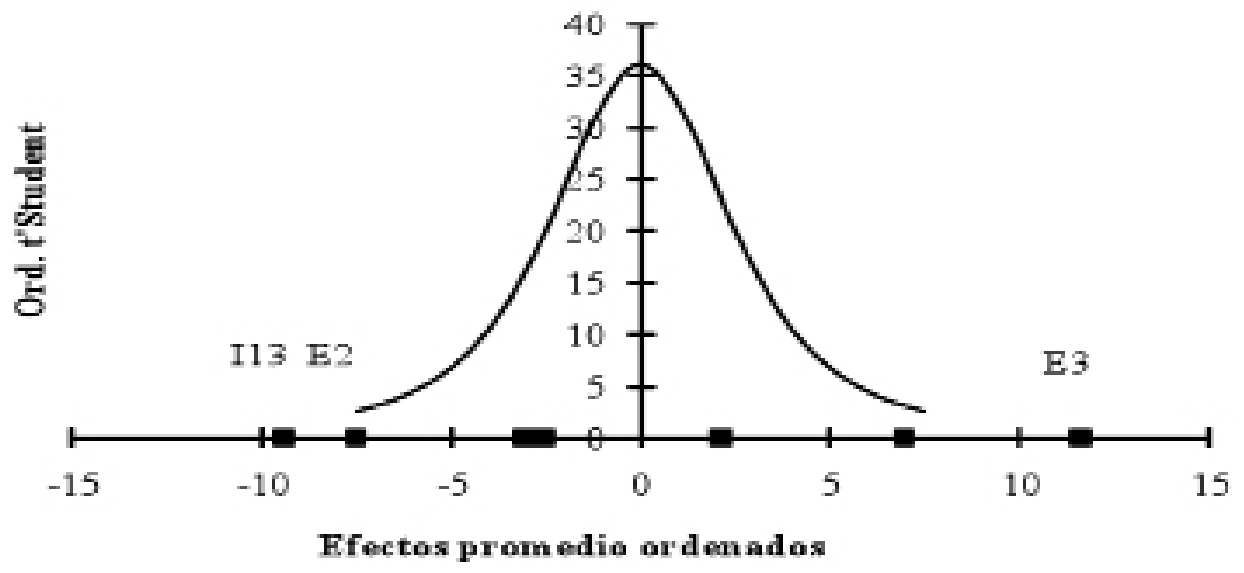

Figura 3. Gráfico t' Student para los efectos e interacciones Fuente: (La autora y los autores) 
Cuadro 4. Análisis estadístico para la variable de conversión de sacarosa en azúcares reductores, segunda etapa experimental

\begin{tabular}{|c|c|}
\hline Estadístico & Valor \\
\hline Promedio, conversión de sacarosa (\%) & 84,74 \\
\hline Efecto 1 , concentración sacarosa, E1 & 6,96 \\
\hline Efecto 2, flujo volumétrico, E2 & $-7,52$ \\
\hline Interacción1-2, I12 & $-3,07$ \\
\hline Efecto 3 , temperatura, E3 & 11,59 \\
\hline Interacción 1-3, I13 & $-9,41$ \\
\hline Interacción 2-3, I23 & 2,11 \\
\hline Interacción 1-2-3, I123 & $-2,53$ \\
\hline Promedio de los efectos & $-0,27$ \\
\hline Varianza de los efectos, $\sigma^{2}$ & 6,16 \\
\hline Intervalo de no significancia (95\%) & {$[-7,44 ; 7,44$} \\
\hline
\end{tabular}

Fuente: (La autora y los autores)

Cuadro 5. Porcentaje de conversión de sacarosa en azúcares reductores a diferentes concentraciones de sustrato a una temperatura de $50^{\circ} \mathrm{C}$, flujo volumétrico de $2,5 \mathrm{~mL} / \mathrm{min}$ y $\mathrm{pH} 4,5$

$\begin{array}{cc}\begin{array}{c}\text { Concentración sacarosa, } \\ \boldsymbol{C n}_{\text {sacarosa }}(\mathbf{m o l} / \mathbf{L})\end{array} & \begin{array}{c}\text { Conversión de sacarosa, } \\ (\%)\end{array} \\ 0,75 & 75,31 \\ 0,85 & 85,47 \\ 1,00 & 94,26 \\ 1,10 & 94,96 \\ 1,20 & 93,12\end{array}$

Fuente: (La autora y los autores)

la desnaturalización de la enzima, además, entre mayor sea la temperatura el consumo de energía es mayor. De esta manera se prefiere trabajar a un nivel bajo de temperatura, en este caso $50^{\circ} \mathrm{C}$ y un nivel alto de concentración $(1,00 \mathrm{~mol} / \mathrm{L})$; con un porcentaje de conversión de $87,13 \%$.

Se prefieren estos niveles ya que a $50^{\circ} \mathrm{C}$ y una concentración de sacarosa $1,00 \mathrm{~mol} / \mathrm{L}$, se obtienen porcentajes altos de conversión. Los resultados obtenidos por Soto (2007), muestran que la actividad de la invertasa se favorece a $50^{\circ} \mathrm{C}$. También se tiene que al trabajar a concentraciones elevadas de sustrato $(1,00 \mathrm{~mol} / \mathrm{L})$ es menor la cantidad de agua que se requiere evaporar para concentrar la sacarosa, lo cual implica un menor consumo energético.

\subsection{Efecto de la concentración inicial de sacarosa}

En la Figura 4 se presenta la variación del porcentaje de conversión de sacarosa en azúcares reductores con el tiempo para distintas concentraciones de sustrato, se observa que conforme aumenta la concentración inicial de sustrato el porcentaje de conversión se incrementa.

En el Cuadro 5 se muestran los resultados de conversión de sacarosa en azúcares reductores, en el estado estable, para diferentes concentraciones de sustrato, dichos valores se encuentran representados en la Figura 5.

Se obtiene que el mayor porcentaje de conversión es del 94,96 \% para una concentración 


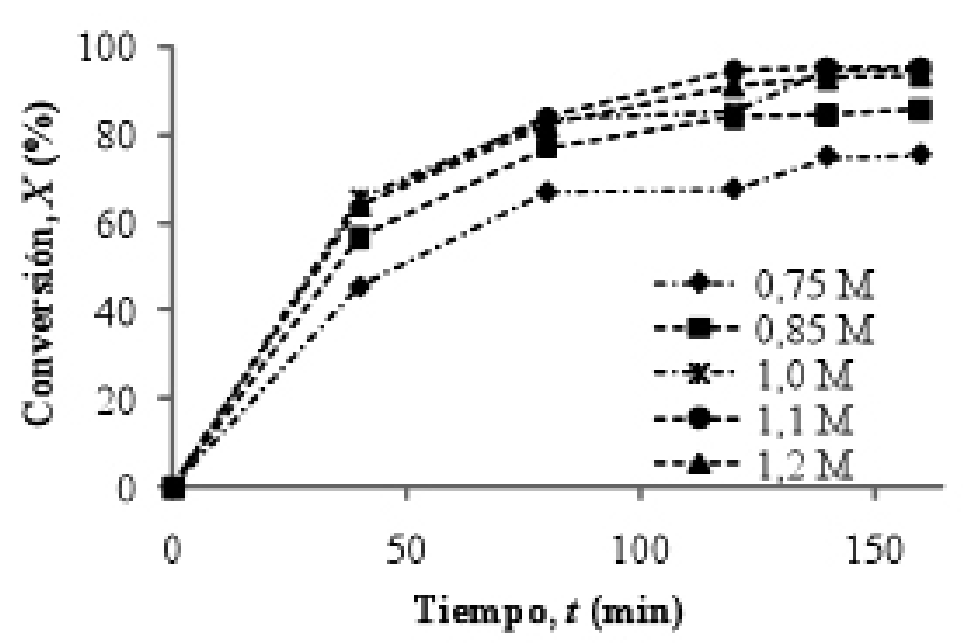

Figura 4. Porcentaje de conversión de sacarosa en azúcares reductores a diferentes concentraciones de sustrato: temperatura de $50^{\circ} \mathrm{C}$, flujo volumétrico de $2,5 \mathrm{~mL} / \mathrm{min}$ y $\mathrm{pH} 4,5$

Fuente: (La autora y los autores)

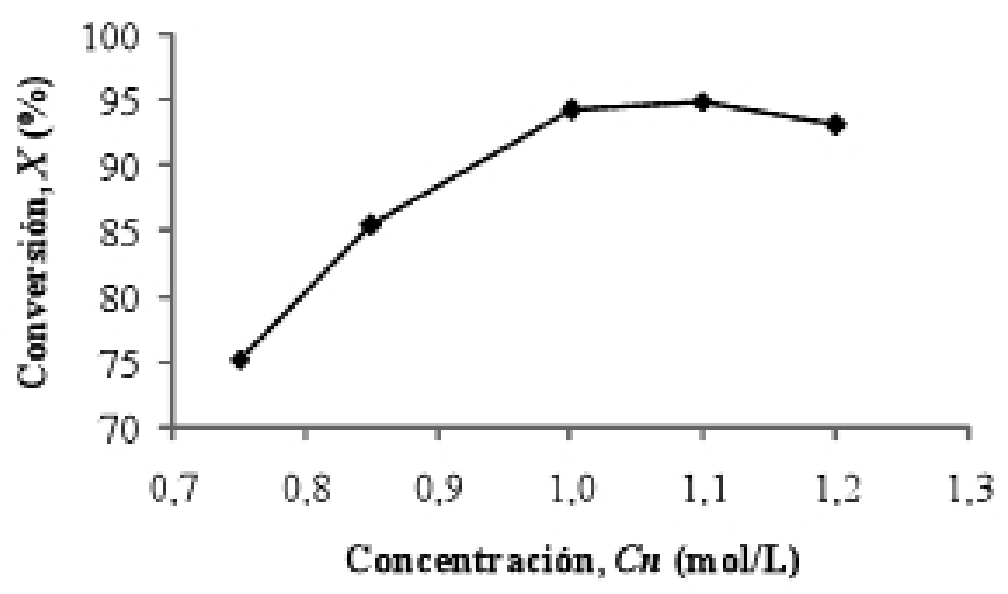

Figura 5. Porcentaje de conversión de sacarosa en azúcares reductores a diferentes concentraciones de sustrato a una temperatura de $50^{\circ} \mathrm{C}$, flujo volumétrico de $2,5 \mathrm{~mL} / \mathrm{min}$ y $\mathrm{pH} 4,5$, en el estado estable Fuente: (La autora y los autores) 
a)

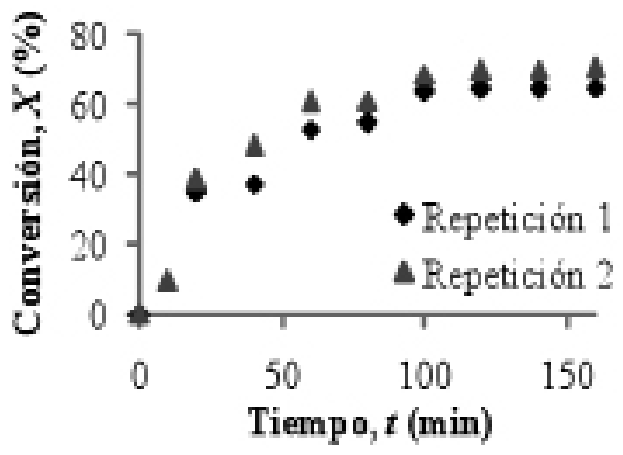

b)

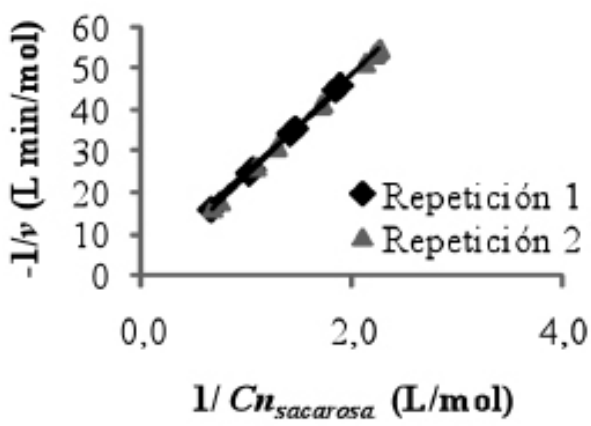

Figura 6. a) Variación del porcentaje de conversión de sacarosa en azúcares reductores b) Gráfico Lineweaver - Burk para la hidrólisis de sacarosa a una concentración 1,5 mol/L, una temperatura de $45^{\circ} \mathrm{C}$ y un flujo volumétrico de $3,0 \mathrm{~mL} / \mathrm{min}$ Fuente: (La autora y los autores)

a)

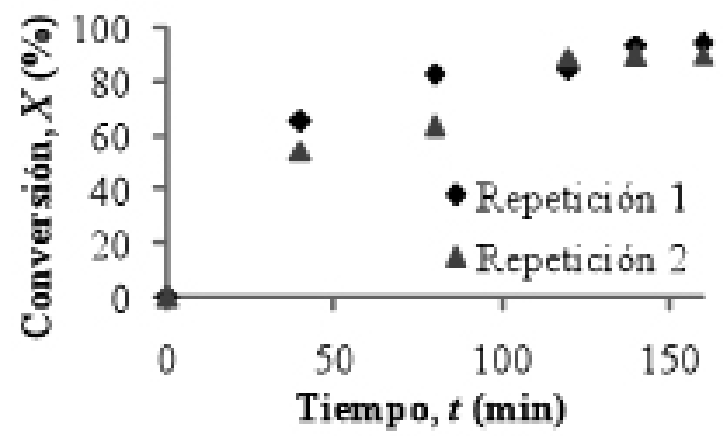

b)

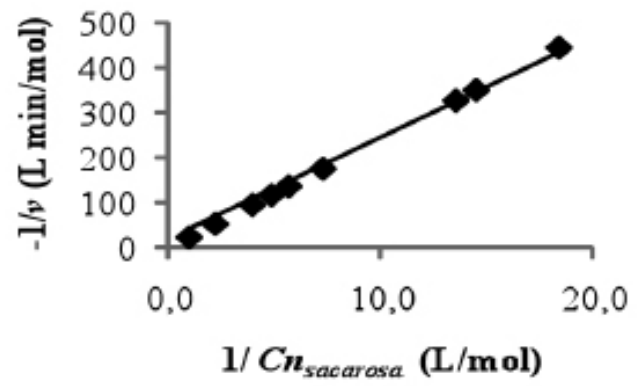

Figura 7. a) Variación del porcentaje de conversión de sacarosa en azúcares reductores

b) Gráfico Lineweaver - Burk para la hidrólisis de sacarosa a una concentración 1,0 mol/L, çuna temperatura de $55^{\circ} \mathrm{C} \mathrm{y} \mathrm{un}$ flujo volumétrico de $3,0 \mathrm{~mL} / \mathrm{min}$ Fuente: (La autora y los autores) 
de $1,1 \mathrm{~mol} / \mathrm{L}$. Además se tiene que conforme aumenta la concentración de sacarosa desde $0,75 \mathrm{~mol} / \mathrm{L}$ hasta $1,1 \mathrm{~mol} / \mathrm{L}$ la conversión se incrementa, pero para una concentración de 1,2 mol/L la conversión empieza a decaer según las condiciones de operación del reactor.

Por lo tanto es preferible trabajar a concentraciones de $1,1 \mathrm{~mol} / \mathrm{L}$ ya que si la concentración es mayor, la presencia de un exceso de sustrato puede actuar como inhibidor enzimático lo cual provoca que la conversión de sacarosa en azúcares reductores disminuya, tal y como pasa con una concentración de 1,2 mol/L. Si es una concentración menor hay que evaporar una mayor cantidad de agua para concentrar la sacarosa, lo cual implica un mayor consumo de energía y un costo económico mayor, además de que el porcentaje de conversión disminuye.

\subsection{Parámetros cinéticos}

Se determinan los parámetros cinéticos de la ecuación de Michaelis - Menten para cada uno de los ensayos, a partir del gráfico de Lineweaver Burk. En las Figuras 6 y 7 se muestra un caso de la variación del porcentaje de conversión con el tiempo y de un gráfico de Lineweaver - Burk.

Los valores de la velocidad máxima de reacción (vmax) y la constante de Michaelis - Menten o constante de afinidad $(\mathrm{Km})$, son específicos para la invertasa así como para las condiciones a las cuales se llevó a cabo la reacción. Los valores

Cuadro 6. Parámetros cinéticos de la ecuación de Michaelis - Menten

Variables

$\begin{array}{ccc}\begin{array}{c}\text { Concentración } \\ \text { sacarosa, }\end{array} & \begin{array}{c}\text { Flujo volumétrico, } \\ \boldsymbol{F} \boldsymbol{v}(\mathbf{m L} / \mathbf{m i n})\end{array} & \begin{array}{c}\text { Temperatura, } \\ \boldsymbol{T}\left({ }^{\circ} \mathbf{C}\right)\end{array} \\ \begin{array}{c}\text { Cn } \boldsymbol{n}_{\text {sacarosa }}(\mathbf{m o l} / \mathbf{L}) \\ 1,00\end{array} & 3,0 & 45 \\ 1,50 & 3,0 & 45 \\ 1,00 & 4,0 & 45 \\ 1,50 & 4,0 & 45 \\ 1,00 & 3,0 & 50 \\ 1,50 & 3,0 & 50 \\ 1,00 & 4,0 & 50 \\ 1,50 & 4,0 & 50 \\ 0,75 & 2,5 & 50 \\ 1,00 & 2,5 & 50 \\ 0,75 & 3,0 & 50 \\ 1,00 & 3,0 & 50 \\ 0,75 & 2,5 & 55 \\ 1,00 & 2,5 & 55 \\ 0,75 & 3,0 & 55 \\ 1,00 & 3,0 & 55 \\ 0,85 & 2,5 & 50 \\ 1,10 & 2,5 & 50 \\ 1,20 & 2,5 & 50 \\ & & \end{array}$

Parámetros

\begin{tabular}{cccc}
$\begin{array}{c}\text { Velocidad } \\
\text { máxima, } \boldsymbol{v}_{\text {max }}\end{array}$ & \multicolumn{2}{c}{ Constante } \\
de Michaelis - \\
(mol/L min) \\
0,13 & - & 2,93 & Menten, $\boldsymbol{K}_{\boldsymbol{m}}$ (mol/L) \\
0,34 & 0,30 & 7,69 & 6,72 \\
0,28 & - & 4,65 & - \\
0,67 & - & 11,11 & - \\
0,05 & - & 1,10 & - \\
0,20 & - & 4,43 & - \\
0,06 & 0,06 & 1,11 & 1,04 \\
0,50 & 0,53 & 8,29 & 9,13 \\
0,10 & - & 2,67 & - \\
0,04 & 0,06 & 1,06 & 1,89 \\
0,16 & - & 3,61 & - \\
0,13 & - & 2,86 & - \\
0,04 & - & 1,09 & - \\
0,04 & - & 1,09 & - \\
0,05 & - & 1,23 & - \\
0,08 & 0,13 & 1,83 & 3,01 \\
0,10 & - & 2,61 & - \\
0,05 & - & 1,28 & - \\
0,07 & - & 1,79 & -
\end{tabular}


de velocidad máxima de reacción se encuentran entre $0,04 \mathrm{~mol} / \mathrm{L}$ min y $0,67 \mathrm{~mol} / \mathrm{L}$ min y los de la constante de afinidad van desde $1,04 \mathrm{~mol} / \mathrm{L}$ hasta $11,11 \mathrm{~mol} / \mathrm{L}$, tal y como se observa en el Cuadro 6.

Los parámetros cinéticos son mayores que los reportados por Soto (2007), quien trabajó con enzima sin inmovilizar. En esta investigación, la inmovilización enzimática provocó un incremento en la velocidad máxima de reacción al comparar con la enzima libre, lo mismo sucede con el parámetro de Michaelis - Menten, lo cual implica que se requiere una mayor concentración de sustrato para alcanzar una misma tasa de reacción con respecto a la enzima libre. Por lo tanto se requiere de una concentración de sustrato mayor para saturar la enzima inmovilizada, esto se da por una disminución en el gradiente de concentración que se produce en los poros de la red polimérica (Woodward, 1985).

Se determina que, conforme la temperatura aumenta se tienen valores del parámetro $\mathrm{Km}$ menores, es decir, existe una mayor afinidad de la enzima por el sustrato que a temperaturas menores.

\section{CONCLUSIONES}

1. Se obtuvo que la cantidad máxima de enzima inmovilizada es de $0,130 \mathrm{mg} / \mathrm{g}$ de soporte a los $220 \mathrm{~min}$.

2. De la primera y segunda etapa experimental se encuentra que, un flujo volumétrico de $2,5 \mathrm{~mL} / \mathrm{min}$, una temperatura de $50^{\circ} \mathrm{C}$ y una concentración inicial de sacarosa $1,0 \mathrm{~mol} / \mathrm{L}$, favorecen la conversión.

3. La máxima conversión que se obtuvo en el estado estable es de un 95,43\%, para una concentración inicial de sacarosa $1,0 \mathrm{~mol} / \mathrm{L}$ un flujo volumétrico de $4 \mathrm{~mL} / \mathrm{min}$ y una temperatura de $50^{\circ} \mathrm{C}$.

4. Al variar la concentración inicial de sacarosa y mantener fija la temperatura en $50^{\circ} \mathrm{C}$ y el flujo volumétrico en $2,5 \mathrm{~mL} / \mathrm{min}$; se encuentra que el mayor porcentaje de conversión es de 94,96 \% para una concentración de 1,1 mol/L.

5. Los valores de velocidad máxima de reacción se encuentran entre $0,04 \mathrm{~mol} / \mathrm{L} \mathrm{min}$ y $0,6 \mathrm{~mol} / \mathrm{L} \mathrm{min}$.

6. Los valores del parámetro de Michaelis - Menten $(\mathrm{Km})$, varían en el intervalo de
$1,04 \mathrm{~mol} / \mathrm{L}$ hasta $11,11 \mathrm{~mol} / \mathrm{L}$, según las condiciones de operación.

7. La utilización de un reactor esférico es una buena opción para la producción de jarabes invertidos y con una alta conversión, ya que se logró mayores conversiones utilizando mayores flujos volumétricos que los reportados por otros investigadores utilizando un reactor tubular.

8. Controlando las condiciones de operación puede aumentarse la conversión.

\section{BIBLIOGRAFÍA}

Amaya-Delgado, L., Hidalgo-Lara, M.E. \& Montes-Horcasitas, M.C. (2006) Hydrolysis of sucrose by invertase immobilized on nylon-6 microbeads. Food Chemistry, 99, 299-304.

Audesirk, T. \& Audesirk, G. (1996). La vida en la Tierra. (4 $\left.4^{\mathrm{a}} \mathrm{ed}\right)$. México: Prentice Hall.

Box, G., Hunter, W. \& Hunter, J.S. (1993). Estadística para investigadores: introducción al diseño de experimentos, análisis de datos y construcción de modelos. Barcelona, España: Reverté.

Bradford, M. (1976). A rapid and sensitive method for the quantitation of microgram quantities of protein utilizing the principle of protein-dye binding. Analytical biochemistry, 72, 248-254.

Fogler, S. (2001). Elementos de ingeniería de las reacciones químicas. ( $3^{\mathrm{a}}$ ed). México, DF: McGraw Hill.

Herrera, C., Bolaños, N. \& Lutz, G. (2003). Química de alimentos: manual de laboratorio. San José, Costa Rica: Editorial Universidad de Costa Rica.

Illanes, A. (1994). Biotecnología de enzimas. Chile: Ediciones Universitarias de Valparaíso de la Universidad Católica de Valparaíso.

Montes, C. (2004). Taller de biotecnología enzimática. Centro de Investigaciones en Productos Naturales (CIPRONA). Universidad de Costa Rica.

Novozymes. (2002). Ficha técnica. Jugos y vegetales. 2002-18896-01.pdf

Ortega, J. (1993). Lactose hydrolysis by immobilized $\beta$-galactosidase on 
nylon-6: a novel spin-basket reactor. Biotechnology Techniques, 7, 775-780.

Salleh,A.(1982). Activation of nynon by alkaline glutaraldehyde solution for enzyme inmovilisation. Biotechlology Letters,vol4 (12) 769-774.

Soto, D. (2007). Evaluación de la reacción de hidrólisis de la sacarosa utilizando una invertasa inmovilizada y sin inmovilizar. Proyecto de graduación para optar por la Licenciatura en Ingeniería Química, Escuela de Ingeniería Química, Universidad de Costa Rica, San José, Costa Rica.

Southgate, D. (1976). Determination of food carbohydrates. London, United Kingdom: Applied Science Publications Ltd.

Woodward, J. (1985). Immobilised cells and enzymes: a practical approach. Oxford, Inglaterra: Irl Press.

\section{SOBRE LOS AUTORES}

\section{María Monserrat Zamora Leitón}

Licenciada en Ingeniería Química de la Universidad de Costa Rica. Funcionaria de Empresa Bridgstone Costa Rica.

\section{Manuel Molina Córdoba}

Ingeniero Químico, Profesor Asociado de la Escuela de Ingeniería Química de la Universidad de Costa Rica.

Correo electrónico:MANUEL.MOLINA@ucr.ac.cr

\section{Gerardo Chacón Valle}

Ingeniero Químico, Profesor Asociado de la Escuela de Ingeniería Química de la Universidad de Costa Rica.

Correo electrónico: GERARDO.CHACON@ ucr.ac.cr 